\title{
Designing Against the Status Quo
}

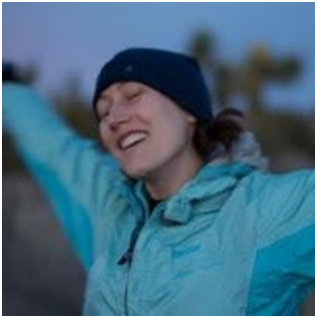

Ellie Harmon is an ethnographer whose work examines the role of

computing technologies - and stories about them - in social life and knowledge-making. She is a Research Associate in the Department of Information Science at the University of Colorado Boulder, studying everyday philanthropic work, with an interest in how to provoke social interactions outside the status quo.

\section{Ellie Harmon \\ Department of Information \\ Science \\ University of Colorado Boulder \\ ellie@ellieharmon.com}

\section{Matthias Korn}

Institute for Information

Systems

University of Siegen, Germany

matthias.korn@uni-siegen.de

\author{
Ann Light \\ School of Engineering and \\ Informatics \\ University of Sussex, UK, \\ ann.light@sussex.ac.uk
}

\section{Amy Voida}

Department of Information

Science

University of Colorado Boulder

amy.voida@colorado.edu

Permission to make digital or hard copies of part or all of this work for personal or classroom use is granted without fee provided that copies are not made or distributed for profit or commercial advantage and that copies bear this notice and the full citation on the first page. Copyrights for third-party components of this work must be honored. For all other uses, contact the Owner/Author.

Copyright is held by the owner/author(s)

DIS '16 Companion, June 04-08, 2016, Brisbane, QLD, Australia

http://dx.doi.org/10.1145/2908805.2913020

\begin{abstract}
In this one-day workshop, we interrogate design strategies of troubling, friction, queering, and contestation that aim to question the status quo. In ways that are playful, heretical, theoretical, and applied we examine tactics that make space for alternative values to emerge in everyday life. Recent design strategies in this space include Light's adaptation of feminist and queer theory in proposing design that troubles and queers the status quo [6], Korn and Voida's adaptation of anthropological theory and theories of the everyday to call for design that causes friction [5], and DiSalvo's formulation of adversarial design as a way of challenging conventional politics [3]. As we design interactive systems that, on the one hand, seek to be accountable in responding to current and future societal challenges, and, on the other, are becoming ever more complex, we ask what trends in destabilizing and rethinking may help us innovate in both method and outcome.
\end{abstract}

\section{Author Keywords}

Queering; troubling; friction; values; politics of design; cultural theory; feminist theory; anthropological theory.

\section{ACM Classification Keywords}

H.5.m. Information interfaces and presentation (e.g., $\mathrm{HCI})$ : Miscellaneous. 


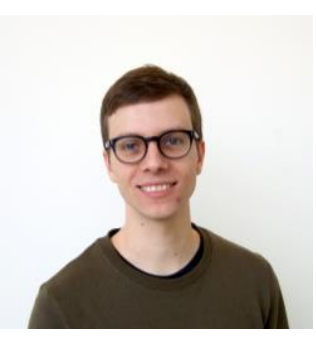

Matthias Korn is a Postdoctoral Researcher at the University of Siegen, Germany. His research interests include studying and designing for civic engagement, activism, subversion, and transgression. He has a background in Information Systems, Human-Computer Interaction, and Participatory Design.

\section{Introduction}

In recent years, there have been several calls for design that aims to trouble and queer [6], create friction [5], or function adversarially [3]. In contrast to user-centered design that aims to make life easier and smooth over apparent gaps and problems in everyday routines, these approaches to design aim to generate friction as a way of drawing attention to invisible infrastructures or disrupting the status quo in the everyday. As Korn and Voida write, in the context of civic engagement, "friction produces movement, action, and effect" and can help to "provoke people not only to take up more active roles in their communities but to question conventional norms and values about what it means to be a citizen as well" [5]. As Light argues, "HCI can begin to tackle gender - and other inequalities, not through attempting to co-opt design to particular ends, but by promoting design which is spaceful, oblique and occasionally mischievous" [6].

We note that the social challenges in which many researchers want to intervene are enmeshed in the ongoing reproduction of society-as-it-already-is. To us, this suggests that design in service of (indeterminately) troubling the routines of the status quo is itself an intervention worth making. Rooted in a diversity of intellectual traditions including feminist and queer theory (e.g., [6] drawing on [2]), anthropological theory and psychology (e.g., [5] drawing on [9] and [4]), and political theory (e.g., [3] drawing on [7]), these tactics offer one response to recent calls for a renewed engagement with the politics and histories of technologies without a prescriptive orientation. Rather than trying to neatly diagnose problems and then propose the correct kind of change/solution in advance, tactics of troubling or creating friction aim to intervene indeterminately in complex situations and create space for alternative values to emerge.

These turbulent approaches to design thus entail a move away from the idea that we should design objects and artifacts that "fit comfortably within everyday routines and augment them without losing or disrupting the qualities that make them what they are" [8]. Instead of promoting stability, they emphasize a livedness to design work, research, and computational artifacts. They "make a space for flexible interactions of the future, rather than stipulate a desired outcome in societal terms" [6]. In this workshop, we are especially interested in thinking about how such orientations and tactics might be taken up in the work of creating everyday and mundane technologies. How can we engage the possibilities of an indirect troubling in our research and design endeavors?

\section{Themes and Participation}

We invite 2-4 page contributions from practitioners and researchers in ACM Extended Abstracts format

(including Pictorials). We also invite other submission formats that embody subversions - of which we cannot (of course) conceive in advance. Ideally all submissions should be in a form that allows sharing via the workshop website in advance of the event. Submissions should be e-mailed to the organizers by April 11, 2016. Key topics of interest include, but are not limited to:

- Theoretical provocations on the design strategies of troubling and/or creating friction for the everyday and mundane

- Discussions/critiques of everyday and mundane artifact(s) or service(s) that embody a problematic 


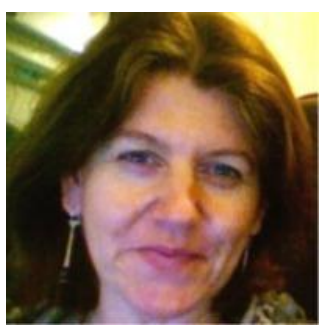

Ann Light is Professor of Design and Creative

Technology and leads the Creative Technology Group at the University of Sussex. She is a researcher specializing in design for social change, bottom-up innovation and the politics of participation. Recent projects have focused on the sociality of urban living as we age, cultures of sharing, and multicultural food growing. She is also a coauthor of "Designing Connected Products: UX for the Consumer Internet of Things" (2015) status quo or neglect/inhibit troubling and/or creating friction

- Design methods and processes that evoke/foster or hinder troubling and/or creating friction as a design strategy for the everyday and mundane

- Empirical/case studies of troubling and/or creating friction at play in the everyday and mundane

\section{Outcomes}

The goal of the workshop is to generate conversation and connections among designers and researchers who use concepts or techniques related to troubling and/or creating friction in their work. The workshop is organized around the production of three concrete outcomes: a design wall; a collection of teaching, design, and research resources; and a report back to the broader design and HCI communities.

During the workshop, we will produce a design wall as a collage that collects together workshop participants' examples of and inspirations for design that seeks to trouble or create friction. Examples of content include: photographs or sketches of objects or artifacts that aim to trouble; sites or situations that need troubling; short quotes offering theoretical elucidation, empirical observations, questions, or provocations. The wall will be produced over the course of the workshop and displayed during the main conference adjacent to the coffee break area (if possible) with an opportunity for all attendees to add comments through the week.

As part of the workshop, we will collectively compile notes with an orientation toward generating a set of resources for teaching, design, and research that will be archived on the workshop website for public use.
We anticipate that this archive would include collections of resources along multiple dimensions, with the final composition informed by workshop participants:

- A provisional elaboration: What does it mean to orient toward design from a perspective of troubling and/or creating friction? What are we turning toward in making such moves? From what are we turning away? Why (when, how) are these techniques needed or useful or generative - or not?

- Bibliography: Collected readings and theoretical resources - a mapping of workshop participants' intellectual genealogies.

- Examples and cases: Objects and artifacts that embody troubling or friction; methods and tactics for troubling or friction; empirical cases, attempts, and outcomes of troubling or friction at play.

- Reinventions and reimaginings: Re-readings of already existing design papers and projects for what is not mentioned, asked, or oriented to.

- Opportunities and open questions: Provocations for future work. What is apparent as missing in the elaboration, bibliography, and cases surfaced and shared at the workshop? Where is new work needed? What are some domains in which this approach could be generative? What are some elements of a research agenda moving forward?

After the workshop, organizers will collaborate on an article that reports back to the broader design community. We anticipate producing something along the lines of an interactions magazine article that would map out some of the various examples of, tactics for, and intellectual genealogies of troubling and creating friction collected at the workshop (e.g., similar to [1]). 


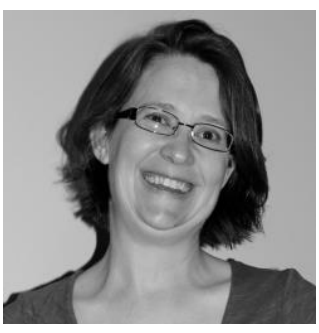

Amy Voida is an Assistant Professor in the Department of Information Science at the University of Colorado

Boulder. She also holds an adjunct appointment with the Lilly Family School of Philanthropy at Indiana University. Her research focuses on understanding the role of technologies in the nonprofit sector and in designing to support and provoke philanthropy and civic engagement.

\section{Workshop Structure}

- Introductions and Genealogies (1 hr)

Participants give 2 minute introductions that respond to the question "How did you get here?"

- Plan for the day, plan for outcomes (30 min) Discuss and augment plan for the day and outcomes.

[ Coffee Break (30 min) ]

- Constructing the design wall (1 hr) As above.

[ Lunch (1.5 hours) ]

- Questioning the wall (45 min)

Open brainstorm, facilitated by questions: What is missing? Where are there clusters and relations? What are the open questions? Where are there resonances and dissonances among contributions?

- Walk \& Talk: Troubling the Everyday (45 min) Walking activity in small groups, practicing troubling, looking for examples of design-in-the-environment, and queering these examples together, reading for what is not mentioned, asked or oriented to.

[ Coffee Break (30 min)]

- Report Back to the Group (30 min)

Share walk conversations; augment design wall.

- Working Groups (30 min)

Self-organize around areas of interest, geared

toward the production of outcomes and next steps, e.g., organizing bibliography for website, collecting cases for website, organizing follow-up activities.

Requested Facilities

In addition to standard workshop space: access to a printer to produce additional materials for the design wall, and space to display the design wall during the conference ( $2 \times 2$ meters, near the coffee break area).

\section{Acknowledgements}

Thanks to Lilly Irani for her comments on an early draft. This material is based upon work supported by the National Science Foundation under Grant Number 1602660. Any opinions, findings, and conclusions or recommendations expressed in this material are those of the author(s) and do not necessarily reflect the views of the National Science Foundation.

\section{References}

1. Baumer, Eric P. S., Jenna Burrell, Morgan G. Ames, Jed R. Brubaker, and Paul Dourish. 2015. On the importance and implications of studying technology non-use. interactions 22, 2. 52-56.

2. Butler, Judith P. 1990. Gender Trouble: Feminism and the Subversion of Identity. Routledge

3. DiSalvo, Carl. 2012. Adversarial Design. MIT Press.

4. Hassenzahl, Marc and Laschke, Matthias. 2015.

"Pleasurable troublemakers". In S.P. Walz \& S. Deterding (Eds.), The Gameful World (ch. 6, pp. 167-195). MIT Press.

5. Korn, Matthias and Amy Voida. 2015. "Creating friction: Infrastructuring civic engagement in everyday life". In Proc. Critical Alternatives: Decennial Aarhus Conference '15. 145-156.

6. Light, Ann. 2011. "HCI as Heterodoxy: Technologies of Identity and the Queering of Interaction with Computers." Interacting with Computers 23: 430-38.

7. Mouffe, Chantal. 2000. The Democratic Paradox. Verso.

8. Tolmie, Peter, James Pycock, Tim Diggins, Allan MacLean, and Alain Karsenty. 2002. "Unremarkable Computing." In Proc. CHI 2002, 399-406.

9. Tsing, Anna L. 2005. Friction: An ethnography of global connection. Princeton University Press. 